〈Back to results $\mid$ <Previous 2 of 25 Next

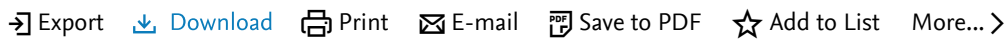

Full Text |View at Publisher|

Document type

Conference Paper

Source type

Conference Proceedings

ISBN

978-172811064-6

DOI

10.1109/ICCCE50029.2021.9467141

View more $\vee$

Proceedings of the 8th International Conference on Computer and Communication Engineering, ICCCE 2021 • Pages 234 - 237 • 22 June 2021 - Article number 9467141 • 8th International Conference on Computer and Communication Engineering, ICCCE 2021 • Kuala Lumpur • 22 June 2021 through 23 June 2021 • Code 171135

\section{Feature Extraction of EEG Signal by Power Spectral Density for Motor Imagery Based BCI}

Alam M.N. 西, Ibrahimy M.I. 四, Motakabber S.M.A. 四

国 Save all to author list

International Islamic University Malaysia, Dpt. of Electrical and Computer Engineering, Kuala, Malaysia

Abstract

Author keywords

Indexed keywords

SciVal Topics

Metrics

Abstract

Signals produced from the brain are widely known as Electroencephalogram (EEG) signal interfacing with any communication device creates a unidirectional communicating channel in the absence of neuro-muscular pathways. An effective Brain-Computer Interface ( $\mathrm{BCI}$ ) system basically consists of three operations which are signal recording, feature extraction and classification. Efficient and reliable classification of EEG signal for motor imagery (MI) based $\mathrm{BCI}$ system depends on the accuracy of denoising and extracted features of the signal. Extracted features are intended to be lossless key information obtained from a signal that describes a dataset accurately. It is important to minimize the classification complexity and maximize the accuracy. Traditional strategies can be used to process the signal, but the diverseness of the EEG signal conceivably could not be depicted utilizing a linear analytical approach. Hence, this paper adopted the power spectral density (PSD) feature extraction technique to extract the features based on various frequency transformations that enhance the classification performance. Graz $\mathrm{BCI}$ competition IV, dataset $2 \mathrm{~b}$ has been utilized in this paper that consisting of two different classes of motor imagery left-hand and right-hand movement. Overall, 0.61 of Cohen's Kappa accuracy obtained using the LDA classifier. @ 2021 IEEE.

Author keywords

$\mathrm{BCI}$; classification; feature extraction; LDA; motor imagery; PSD
Cited by 0 documents

Inform me when this document is cited in Scopus:

Set citation alert >

Related documents

Brain-Computer Interface controlled functional electrical stimulation system for paralyzed arm

Abduallatif, N.A. , Elsherbini, S.G. , Boshra, B.S.

(2017) 2016 8th Cairo International Biomedical Engineering Conference, CIBEC 2016

EEG Features Extraction and Classification Methods in Motor Imagery Based Brain Computer Interface

Malass, M. , Tabbal, J. , El Falou, W. (2019) International Conference on Advances in Biomedical Engineering, ICABME

Electroencephalography (EEG)-based epileptic seizure prediction using entropy and K-nearest neighbor (KNN) Ibrahim, S. , Djemal, R. , Alsuwailem, (2017) Communications in Science and Technology

View all related documents based on references

Find more related documents in Scopus based on:

Authors > Keywords > 


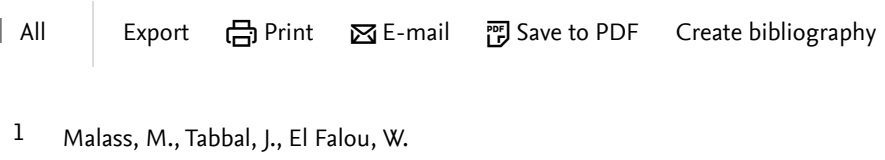

EEG Features Extraction and Classification Methods in Motor Imagery Based Brain Computer Interface

(2019) International Conference on Advances in Biomedical Engineering, ICABME, 2019October, art. no. 8940251.

https://ieeexplore.ieee.org/xpl/mostRecentlssue.jsp?punumber=8119420

ISBN: 978-172812314-1

doi: 10.1109/ICABME47164.2019.8940251

View at Publisher

2 Fang, Y., Chen, M., Zheng, $X$.

Extracting features from phase space of EEG signals in brain-computer interfaces

(2015) Neurocomputing, 151 (P3), pp. 1477-1485. Cited 41 times.

www.elsevier.com/locate/neucom

doi: 10.1016/j.neucom.2014.10.038

View at Publisher

3 Hasan, M.R., Ibrahimy, M.I., Motakabber, S.M.A., Shahid, S.

Classification of multichannel EEG signal by single layer perceptron learning algorithm

(2014) Proceedings - 5th International Conference on Computer and Communication Engineering: Emerging Technologies via Comp-Unication Convergence, ICCCE 2014, art. no. 7031650 , pp. $255-257$

ISBN: 978-147997635-5

doi: $10.1109 /$ ICCCE.2014.79

View at Publisher

4 Hasan, M.R., Ibrahimy, M.I., Motakabber, S.M.A., Shahid, S.

Classification of Multichannel EEG Signal by Linear Discriminant Analysis

(2015) Advances in Intelligent Systems and Computing, 1089, pp. 279-282. Cited 15 times. http://www.springer.com/series/11156

ISBN: 978-331908421-3

doi: 10.1007/978-3-319-08422-0_42

View at Publisher

5 Ibrahim, S., AlSharabi, K., Djemal, R., Alsuwailem, A.

An adaptive learning approach for EEG-based computer aided diagnosis of epilepsy

(2016) Proceeding - 2016 International Seminar on Intelligent Technology and Its Application, ISITIA 2016: Recent Trends in Intelligent Computational Technologies for Sustainable Energy, art. no. 7828633, pp. 55-60. Cited 4 times.

ISBN: 978-150901709-6

doi: $10.1109 /$ ISITIA.2016.7828633

View at Publisher

6 Ivanović, M., Budimac, Z., Radovanović, M., Kurbalija, V., Dai, W., Bădică, C., Colhon, M., (...), Mitrović, D.

Emotional agents - state of the art and applications (Open Access)

(2015) Computer Science and Information Systems, 12 (4), pp. 1121-1148. Cited 16 times. http://www.comsis.org/pdf.php?id=raips-1 doi: 10.2298/CSIS141026047I

View at Publisher 
7 Hassan, A.R., Bhuiyan, M.I.H.

An automated method for sleep staging from EEG signals using normal inverse Gaussian parameters and adaptive boosting

(2017) Neurocomputing, 219, pp. 76-87. Cited 77 times.

www.elsevier.com/locate/neucom

doi: 10.1016/j.neucom.2016.09.011

View at Publisher

8 Tharwat, A., Gaber, T., Ibrahim, A., Hassanien, A.E.

Linear discriminant analysis: A detailed tutorial (Open Access)

(2017) Al Communications, 30 (2), pp. 169-190. Cited 200 times.

http://www.iospress.nl/

doi: 10.3233/AIC-170729

View at Publisher

9 Ivaylov, I., Lazarova, M., Manolova, A.

EEG Classification for Motor Imagery Mental Tasks Using Wavelet Signal Denoising

(2020) 28th National Conference with International Participation, TELECOM 2020

Proceedings, art. no. 9299532 , pp. 53-56.

http://ieeexplore.ieee.org/xpl/mostRecentlssue.jsp?punumber=9299508

ISBN: 978-172818717-4

doi: 10.1109/TELECOM50385.2020.9299532

View at Publisher

10 Tabbal, J., Mechref, K., El-Falou, W.

Brain Computer Interface for smart living environment

(2019) 2018 9th Cairo International Biomedical Engineering Conference, CIBEC 2018 -

Proceedings, art. no. 8641827, pp. 61-64. Cited 5 times.

http://ieeexplore.ieee.org/xpl/mostRecentlssue.jsp?punumber=8637586

ISBN: 978-153868154-1

doi: $10.1109 / C I B E C .2018 .8641827$

View at Publisher

11 Amjed, S., Ausilah, A., Al-Fraihat, A.

Methods of eeg signal features extraction using linear analysis in frequency and timefrequency domains

(2014) International Scholarly Research Notices. Cited 186 times.

Article ID 730218, 7 pages 2014

https://doi.org/10.1155/2014/730218

C) Copyright 2021 Elsevier B.V., All rights reserved.

\section{About Scopus}

What is Scopus

Content coverage

Scopus blog

Scopus API

Privacy matters

\section{Language}

日本語に切り替える

切换到简体中文

切換到繁體中文

Русский язык
Customer Service

Help

Contact us 